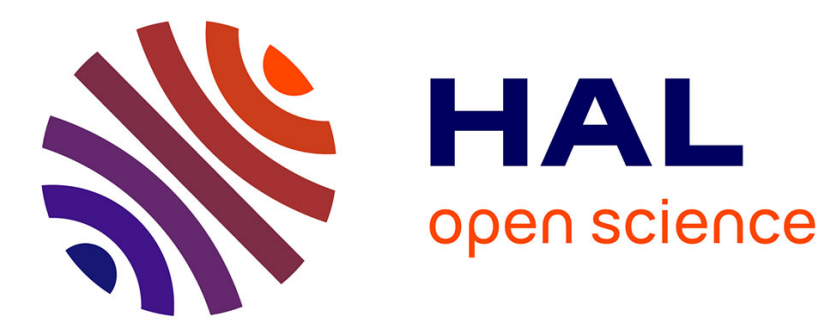

\title{
Loss separation in soft magnetic composites
}

Olivier de La Barrière, Carlo Appino, Fausto Fiorillo, Ragusa Carlo, Hamid Ben Ahmed, Mohamed Gabsi, Frédéric Mazaleyrat, Martino Lobue

\section{To cite this version:}

Olivier de La Barrière, Carlo Appino, Fausto Fiorillo, Ragusa Carlo, Hamid Ben Ahmed, et al.. Loss separation in soft magnetic composites. Journal of Applied Physics, 2011, 109, pp.07A317. hal00584717

\section{HAL Id: hal-00584717 https://hal.science/hal-00584717}

Submitted on 27 Apr 2011

HAL is a multi-disciplinary open access archive for the deposit and dissemination of scientific research documents, whether they are published or not. The documents may come from teaching and research institutions in France or abroad, or from public or private research centers.
L'archive ouverte pluridisciplinaire HAL, est destinée au dépôt et à la diffusion de documents scientifiques de niveau recherche, publiés ou non, émanant des établissements d'enseignement et de recherche français ou étrangers, des laboratoires publics ou privés. 


\section{Loss separation in soft magnetic composites}

6

7

8

9

10

11

O. de la Barrière ${ }^{1}$, C. Appino ${ }^{2}$, F. Fiorillo², C. Ragusa ${ }^{3}$, H. Ben Ahmed ${ }^{1}$,

M. Gabsi ${ }^{1}$, F. Mazaleyrat $^{1}$, M. LoBue ${ }^{1}$
12

13

14

15

16

17

18

19

20
${ }^{1}$ SATIE, ENS Cachan, CNRS, UniverSud, 61 av du President Wilson, F-94230 Cachan, France ${ }^{2}$ Istituto Nazionale di Ricerca Metrologica (INRIM), Strada delle Cacce 91, 10135 Torino, Italy ${ }^{3}$ Dipartimento di Ingegneria Elettrica, Politecnico di Torino, C.so Duca degli Abruzzi 24, 10129 Torino, Italy 
26 We report and discuss significant results on the magnetic losses and their frequency dependence 27 in soft magnetic composites. Two types of bonded Fe-based materials have been characterized at 28 different inductions from DC to $10 \mathrm{kHz}$ and analyzed by extending the concept of loss separation 29 and the related statistical theory to the case of heterogeneous materials. Starting from the 30 experimental evidence of eddy current confinement inside the individual particles, the classical loss 31 component is calculated for given particle size distribution. Taking then into account the 32 contribution of the experimentally determined quasi-static (hysteresis) loss, the excess loss 33 component is obtained and quantitatively assessed. Its behavior shows that the dynamic 34 homogenization of the magnetization process with frequency, a landmark feature of magnetic 35 laminations, is restrained in these materials. This results into a partial offset of the loss advantage 36 offered by the eddy current confinement.

PACS numbers: $75.50 . \mathrm{Bb}$

39 


\section{INTRODUCTION}

43 Three-dimensional flux paths and high frequencies are ubiquitous in modern electrical machines

$44[1][2]$ and laminated cores may often prove inadequate. In fact, while inhibited from out-of-plane magnetization, soft magnetic laminations show a rapid increase of the power loss and fall into skin effect phenomena in the presence of harmonics and/or high working frequencies [3] [4]. Soft magnetic composites (SMCs) are therefore often proposed as convenient substitutes, their use appearing especially suited for devices like permanent magnet, claw-pole, and axial flux machines. While many literature efforts in these materials have been devoted to preparation methods and microstructural studies, with the aim of improving their manufacturing process, their mechanical

51 properties, and their magnetic behavior[5][6], the analysis of the magnetic properties has mostly been performed on qualitative grounds [7][8], aiming at rapid exploitation in SMC machine core modeling [9]. For example, the important problem of loss calculation in such materials has been approached by use of polynomial interpolation methods [10][11], which are not accurate enough to predict the loss under highly distorted induction waveforms. De Wulf, et al. [12] have resorted to the concept of loss separation and attempted to apply Bertotti's statistical theory [13] to the frequency dependence of the energy loss in different types of SMCs. Their study was, however,

58 limited to the maximum frequency of $100 \mathrm{~Hz}$ and their calculations disregard the actual heterogeneous structure of the material.

In this paper we present significant new results on the energy losses and their frequency dependence (DC $-10 \mathrm{kHz}$ ) in commercial soft Fe bonded composites. The experiments show that the sample cross-sectional area negligibly affects the loss at all frequencies. The classical loss component is therefore calculated for eddy current paths confined within the grains and the loss decomposition, leading to the determination of the hysteresis and excess losses, is accordingly performed . The excess loss is observed to depend on frequency in a quasi-linear fashion, in contrast with the square-root law usually observed in magnetic laminations. This provides evidence for correspondingly weakened dynamic homogenization of the magnetization process, ensuing from 
combination of strong internal demagnetizing effects and lack of long-range eddy currents.

\section{EXPERIMENTAL RESULTS AND DISCUSSION}

Two commercial SMCs (Somaloy Prototyping and Somaloy 110i1P) have been investigated. The first one (Material A) has the following physical properties: density $\delta=7450 \mathrm{~kg} / \mathrm{m}^{3}$, electrical resistivity $\rho=2.80 \cdot 10^{-4} \Omega \mathrm{m}$, average particle size $\langle s\rangle=114 \mu \mathrm{m}$. The second material (B) has quite smaller particle size $(\langle s\rangle=29.5 \mu \mathrm{m})$ and lower filling factor (density $\delta=7260 \mathrm{~kg} / \mathrm{m}^{3}$, electrical resistivity $\left.\rho=76 \cdot 10^{-4} \Omega \mathrm{m}\right)$. Ring samples of mean diameter $50 \mathrm{~mm}$ and $25 \mathrm{~mm}$ were prepared for magnetic testing. The larger rings had cross-sectional area $S_{1}=5 \times 5 \mathrm{~mm}^{2}$, the smaller ones $S_{2}=2.5$ $\times 2.5 \mathrm{~mm}^{2}$. Hysteresis loop and loss measurements under sinusoidal induction were carried out, starting from quasi-static conditions ( $5 \mathrm{~Hz}$ for $\mathrm{A}$ and $20 \mathrm{~Hz}$ for $\mathrm{B}$ ), up to $f=10 \mathrm{kHz}$ for peak polarization values $J_{\mathrm{p}}=0.50,1.0,1.25 \mathrm{~T}$. A broadband hysteresisgraph-wattmeter with digital control of the induction waveform endowed with a 5kVA DC-20 kHz CROWN power amplifier was employed. Sample heating was prevented by keeping the sample immersed in a water bath.

A remarkable outcome of these experiments is that the energy loss $W(f)$ does not depend, at all frequencies, on the cross-sectional area of the sample (Fig. 1, SMC type A). The same occurs in the SMC type B. This finding implies that grain-to-grain conductivity phenomena, either due to random metallic contacts through the binder [14] or capacitive effects at high frequencies can be disregarded from the viewpoint of losses. With the eddy current paths confined within the particles, a good simplification is introduced in the loss analysis, because one can concentrate on the behavior of the individual particles and their statistics. To start with, we calculate the classical loss component $W_{\mathrm{cl}}$, which in this case is the contribution that would originate if all particles were magnetized at the same uniform rate imposed to the sample as a whole. Scanning electron microscope images permit one to appreciate size and morphology of the particles and their distribution. The cross-sectional area $S_{\text {part }}$ of each particle is determined from a number of 
micrographs using an image processing software. Their shape is irregular (Fig. 2), but, for the sake of calculation, they are approximated as rectangles. The width and length of these equivalent rectangles are identified by performing extended micrographic analysis and by taking the mean values of particle area and aspect ratio. The Poisson equation for the magnetic field is then solved by a finite element procedure, [12] in order to compute the eddy current loss in the rectangles. The results for materials A and B are shown in Figs. 3 and 4, respectively, where the decomposition of the measured loss W(f) into the hysteresis Whyst and excess Wexc(f) loss components is put in evidence. Whyst is an experimental quantity, obtained by extrapolating the measured $\mathrm{W}(\mathrm{f})$ to $\mathrm{f}=0$. The strong reduction of Wcl(f) ensuing from the reduction of the particle size is apparent. By associating it to a concurring reduction of Wexc(f), one gets that, in spite of higher Whyst, the total loss W(f) in the fine-grained SMC type B becomes lower than in the coarse-grained SMC type A beyond about $2 \mathrm{kHz}$.

As known, the excess loss arises because the dynamic magnetization process, like the quasi-static one, is inhomogeneous in nature. However, since the eddy-current counterfields become stronger with increasing frequency and they have to be compensated by the applied field in order to keep a given magnetization rate $\dot{p}_{\mathrm{p}}$, progressive homogenization of this process is concurrently expected. This concept is made quantitative in terms of active magnetic objects (MOs) and their frequency dependence by the statistical theory of losses [13], which provides the following general expression for the excess loss

$$
W_{e x c}\left(J_{p}, f\right)=2 n_{o} V_{o} J_{p}\left(\sqrt{1+\frac{16 \sigma G S V_{o}}{n_{o}^{2} V_{o}^{2}} f J_{p}}-1\right)
$$

where $G=0.1356, S$ is the cross-sectional area of the sample, $V_{\mathrm{o}}$ is a parameter related to the distribution of the local pinning fields for the MOs, and $n_{0}$ is the number of MOs that are simultaneously active within the sample cross-section under quasi-static magnetization. It is generally found in magnetic laminations that $n_{0}$ is at most a few units [13]. A basic tenet of the theory is that the number of active MOs varies with frequency according to the linear law $n=n_{0}+$ 
$118 H_{\text {exd }} / V_{0}$, where $H_{\text {exc }}=W_{\text {exc }} / 4 J_{\mathrm{p}}$ is the excess field to be dynamically applied in order to counteract 119 the mesoscopic (i.e. circulating close to the moving domain walls) eddy current fields. By taking the experimental $W_{\text {exc }}(f)$, as obtained by subtracting $W_{\text {hyst }}+W_{\mathrm{cl}}(f)$ from the measured $W(f)$ values (see

121 Figs. 3 and 4), the $n$ versus $H_{\text {exc }}$ behavior is obtained, as shown for the A and B type SMCs in Fig. 5 122 for $J_{p}=1 \mathrm{~T}$. The experimental $n_{0}$ and $V_{\mathrm{o}}$ values found in this way are introduced in Eq. (1) and the 123 so calculated $W_{\text {exc }}(f)$, added to $W_{\text {hyst }}$ and the previously obtained $W_{\text {cl }}(f)$, results into the fitting loss 124 curve for $W(f)$ shown in Figs. 3 and 4. A striking result of this analysis is that n0 is orders of 125 magnitude larger than in soft magnetic laminations. $n_{0}$ is a quantity that refers to the whole crosssectional area of the sample [13] and it appears here that the magnetization process in SMCs tends to simultaneously invest a large number of particles at the same time already at low frequencies. It can therefore be assumed that there should be at least one active magnetic object per particle at zero 129 excess field (i.e. under quasi-static excitation). If we take the type A SMC sample with $\mathrm{S}=25 \mathrm{~mm} 2$ and average grain-size area $<\mathrm{s}>2=0.0130 \mathrm{~mm} \mathrm{2}$, one gets the mean number of particles $<$ npart $>$ in

131 the sample cross section <npart> $=1924$, close to the number n0 of MOs (Fig. 5). For this material, 132 the observed dependence of $\mathrm{n}$ on the excess field (i.e. frequency), shown for Jp $=1.0 \mathrm{~T}$ in Fig. 5, 133 suggests that each particle should contain a few MOs, which will be dynamically activated on 134 increasing the frequency. The type B SMC shows a remarkably higher no value, as expected from a 135 correspondingly lower grain size. In both cases we observe that the condition no $>$ Hexc/Vo is approximately satisfied in a good part of the investigated frequency range. A first order expansion 137 of Eq. (3) correspondingly provides Wexc $\propto$ f. This contrasts with usual Hexc/Vo $>>$ no condition 138 already found at low frequencies in magnetic laminations [13], which leads to the typical Wexc $\propto$ $139 \mathrm{f} 1 / 2$ law.

\section{CONCLUSIONS}

The measurement of magnetic losses from $\mathrm{DC}$ to $10 \mathrm{kHz}$ in commercial soft magnetic 142 composite materials and the analysis of the results -centered on the concept of loss decomposition143 highlights peculiar features of these materials. It is verified that the eddy currents are by and large 
144 hindered from grain-to-grain circulation at all frequencies, with ensuing benefits from the viewpoint 145 of energy dissipation. This fact permits one to simplify the problem of loss calculation, whereby the 146 classical loss component can be analytically derived from knowledge of the distribution of the 147 particle size. A general formulation for the excess loss component, as provided by the statistical 148 theory of losses, can then be applied and shown to describe the experimental results and their 149 dependence on frequency. It is induced that the magnetization process in SMCs is characterized by 150 a good degree of homogenization already at very low magnetizing frequencies. The lack of 151 macroscopic eddy current patterns and the related counterfields eventually results, however, into a 152 faster than usual increase of the excess loss component with frequency and into its correspondingly 153 good contribution to the total loss up to the maximum investigated frequency of $10 \mathrm{kHz}$. 
155 156 157 158 159 160 161 162 163 164 165 166 167 168 169 170 171 172 173 174 175 176 177 178 179 180 181 182 183 184
[1] Y. Guo, J. Zhu, Z. Lin, Z., H. Lu, X. Wang, and J. Chen, J. Appl. Phys., 103, $07 F 118$ (2008).

[2] O. Bottauscio, A. Canova, M. Chiampi, and M. Repetto, IEEE Trans. Magn., 38, 805 (2002).

[3] F. Fiorillo and A. Novikov, IEEE Trans.Magn., 26, 2904 (1990).

[4] C. Appino, G. Bertotti, D. Binesti, O. Bottauscio, M. Chiampi, J.P. Ducreux, F. Fiorillo, M. Repetto, and P. Tiberto, J. Appl. Phys., 79, 4575 (1996).

[5] C.G. Oliver, IEEE Trans.Magn., 31, 3982 (1995).

[6] J.A. Bas, J.A. Calero, and M.J. Dougan, J. Magn. Magn. Mater. 254-255, 391 (2003).

[7] T. Kadomura, K. Ishiyama, and M. Yagi, IEEE Trans.Magn., 45, 4294 (2009).

[8] C. Cyr, P. Viarouge, S. Clénet, and J. Cros, IEEE Trans. Magn. 451178 (2009).

[9] Z. Zhang, F. Profumo, A. Tenconi, and M. Santamaria, IEEE Trans. Magn. 334194 (1997).

[10] Y.G. Guo, J.G. Zhu, J. Zhong, J. Magn. Magn. Mater. 302, 14 (2006).

[11] L. Lefebvre, S. Pelletier, and C. Gélinas, J. Magn. Magn. Mater. 76, 93 (1997).

[12] M. De Wulf, L. Anestiev, L. Dupré, L. Froyen, and J. Melkebeek, J. Appl. Phys. 91, 7845 (2002).

[13] G. Bertotti, IEEE Trans. Magn. 24, 621 (1988).

[14] Nord, G., Pennander, L.O., Jack, A., in: Recent Developments of Electrical Drives (Springer, 2004), p. 433. 
187 Fig. 1: Energy loss versus frequency measured at peak polarization values $J_{\mathrm{p}}=0.5 \mathrm{~T}, 1.0 \mathrm{~T}, 1.25 \mathrm{~T}$ in the 188 type A soft magnetic composite $\left(\delta_{\text {proto }}=7450 \mathrm{~kg} / \mathrm{m}^{3}\right)$. The results are obtained on two ring samples of cross189 sectional areas $S_{1}=5 \times 5 \mathrm{~mm}^{2}$ and $S_{2}=2.5 \times 2.5 \mathrm{~mm}^{2}$, respectively.

190 Fig. 2: Micrograph for the type B SMC. The classical eddy current loss component Wcl(f) is calculated 191 assimilating this structure with an array of rectangular grains.

192 Fig. 3: DC-10 kHz loss separation at $J_{\mathrm{p}}=1.0 \mathrm{~T}$ in the SMC type A. The energy loss $W(f)$ (open symbols) is 193 measured under controlled sinusoidal flux. The continuous fitting line is obtained by adding to the 194 experimental hysteresis loss $W_{\text {hyst }}$ the classical loss $W_{\mathrm{cl}}(f)$, calculated with finite elements.

195 Fig. 4: Same as Fig. 3 for the SMC type B.

196 Fig. 5: Number of active magnetic objects $n$ versus the excess field $H_{\text {exc }}=W_{\text {exc }} / 4 J_{\mathrm{p}}$ at $J_{\mathrm{p}}=1.0 \mathrm{~T}$ in the two 197 investigated SMCs. The straight lines provides best fitting with the predicted relationship $n=n_{0}+H_{\text {exd }} / V_{0}$. 


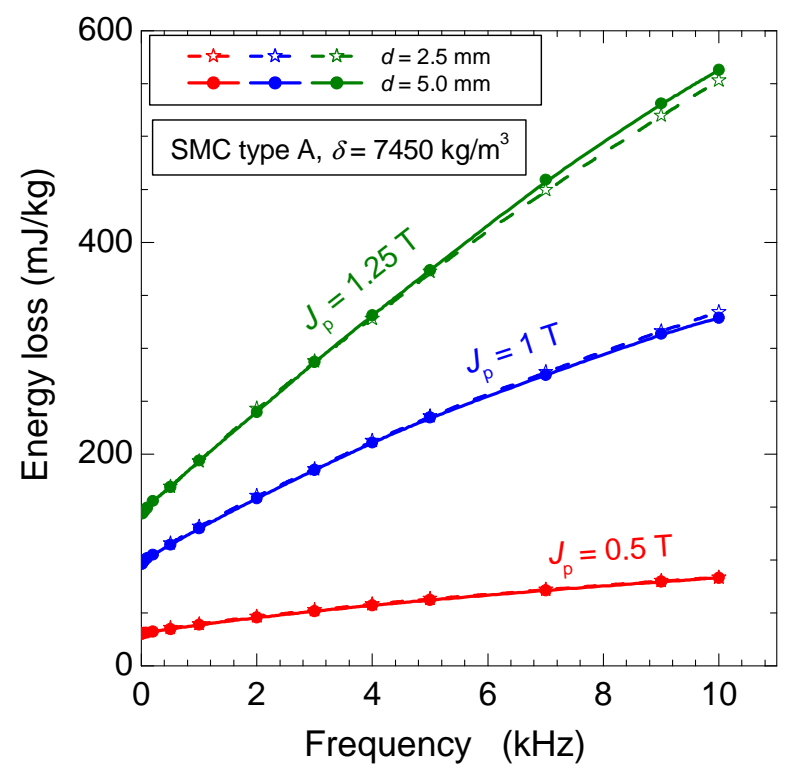

199

200

Fig. 1

201 


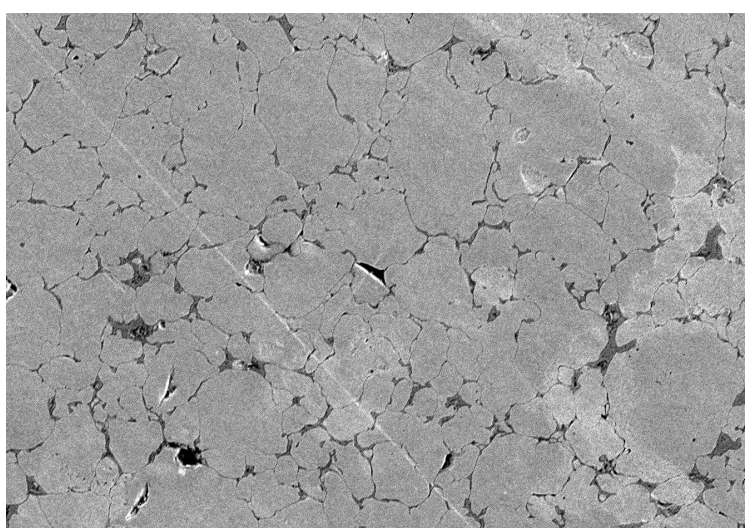

20.0kV $16.1 \mathrm{~mm} \times 320 \mathrm{SE}$

204

Fig. 2 


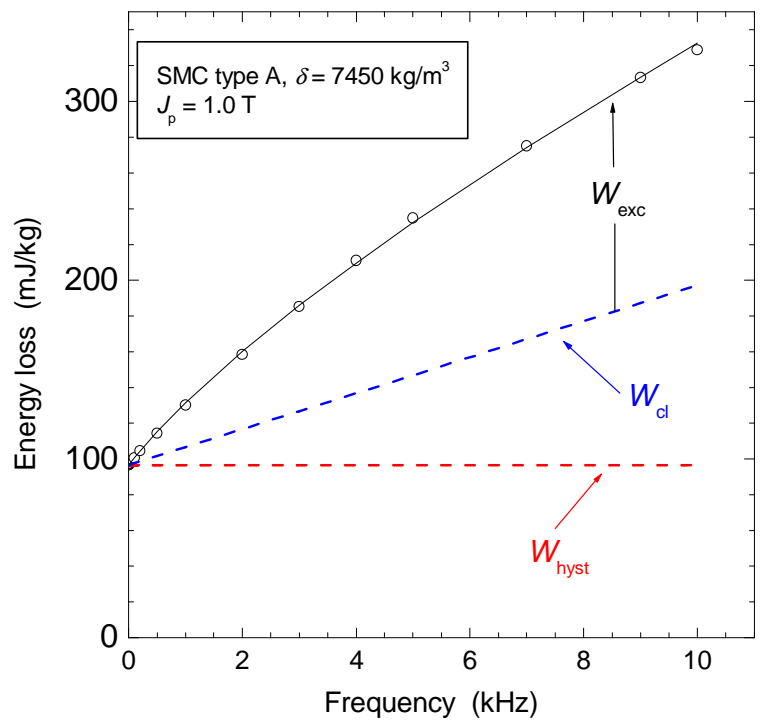

205

206

Fig. 3 


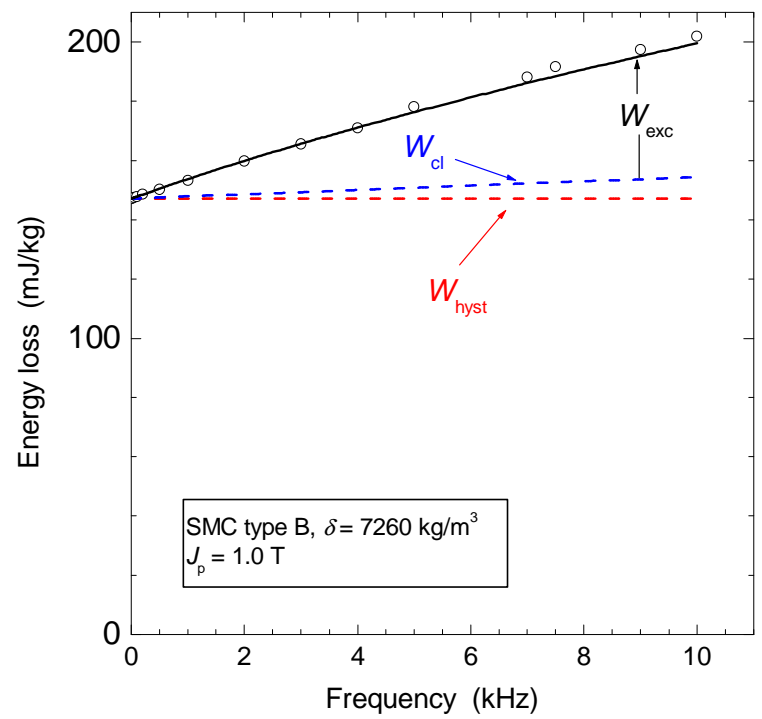

208

209

Fig. 4

210 


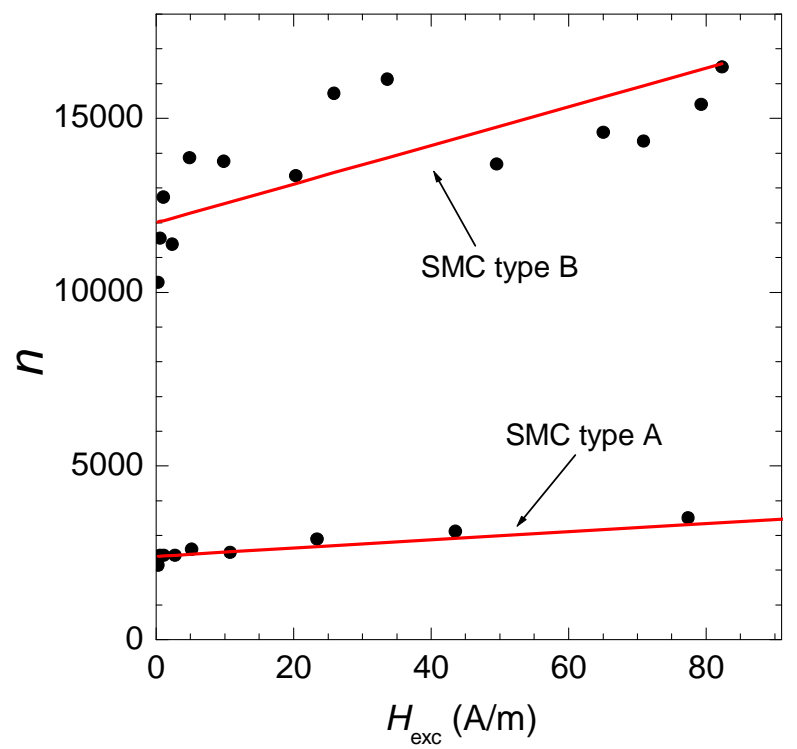

211

Fig. 5 